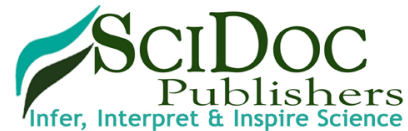

International Journal of Clinical \& Experimental Otolaryngology (IJCEO) ISSN 2572-732X

\title{
Idiopathic Subglottic Stenosis: Surgical and Therapeutic Results in an Adult Woman
}

Research Article

Radhakrishnan $\mathrm{N}^{1 *}$, Mathisen $\mathrm{DJ}^{2}$

${ }^{1}$ Assistant Professor, Department of Speech and Hearing Sciences, Lamar University, Beaumont, Texas, USA.

${ }^{2}$ Professor of Surgery, Harvard Medical School, Chief of Thoracic Surgery, Massachusetts General Hospital, Fruit Street, Blake, Boston, MA, USA.

\section{Abstract}

Introduction: Subglottic stenosis is a condition leading to narrowing of subglottal space due to intubation, inflammation, tracheostomy, trauma, and idiopathic conditions. Respiratory effort of the patient increases due to this narrowing. The effect is also perceived in speech production as effortful exhalation, unnatural pause due to shallow breathing, and muscle tension. Surgical treatment including cricoplasty and tracheal anastomosis can improve respiratory function but adversely affect voice production, especially in adult women. The following case study is a retrospective voice analysis of an adult female before and after cricoplasty and voice therapy.

Methodology: Forty seven year female, diagnosed with Idiopathic subglottic stenosis, was seen pre and post-tailored cricoplasty. The patient underwent eighteen sessions of voice therapy. Pre-post-operative and post-therapy measures of Voice Handicap Index-10, Consensus Auditory Perceptual Evaluation - Voice, fundamental frequency, speaking fundamental frequency, perturbation measures like jitter, and shimmer were compared after therapy.

Results: All the above mentioned voice measures were affected negatively after surgery; however, the patient showed substantial improvement after therapy. Transgender therapy approach helped considerably in this improvement.

Conclusions: This is a single case study. The authors suggest that considering voice therapy before and after surgery may improve prognosis of patients' voice and satisfaction of treatment.

\section{Introduction}

Subglottic stenosis, grouped under laryngotracheal stenosis is a life-threatening condition that restricts pulmonary ventilation. [1] Idiopathic subglottic stenosis (ISS) is a condition that is not associated with history of laryngotracheal injury, intubation, tracheostomy, autoimmune disorders, or iatrogenic incidents. ISS is arare, slowly progressive disorder that is limited to the cricoid and the first two tracheal rings, and mostly seen in Caucasian women between ages 30 and 50 years [2]. Surgery is the highly sought after intervention due to the threat to breathing. Innovations in surgical techniques have improved the prognosis of patients with ISS.

Cricotracheal resection (CTR) and tailored cricoplasty (TC) are some of the most commonly used surgical approach. Though the primary concern reported by patients is dyspnea before surgical intervention, patients do experience voice related problems due to the proximity of the vocal folds and laryngeal structures to thestenosis. Sixty four percent of the patient population that underwent CTR reported inability to project their voice or sing compared to their pre-surgical status [3]. Effect of surgical intervention has the potential to alter voice production in these patients, especially adult females. Cricotracheal anastomosis may lower the larynx from its pre-surgical position and cricoplasty can also affect intrinsic laryngeal muscles attached to the cricoid. Smith et al (2008) [4] reported low pitched voice, below $165 \mathrm{~Hz}$ and decreased phonatory frequency range by 5.9 semitones in fourteen females who underwent CTR for ISS. Similar results were reported later for patients who underwent CTR $[5,6]$. Efficacy of voice therapy has not been reported in the literature for such patients. This is a case-study of a patient who underwent tailored cricoplasty [7] as a surgery approach for ISS.

\section{Case $\mathbf{X}$}

Case X, female, 47 years, diagnosed with ISS was seen at the Voice Lab and Vocology Clinic at Lamar University, Beaumont, Texas.

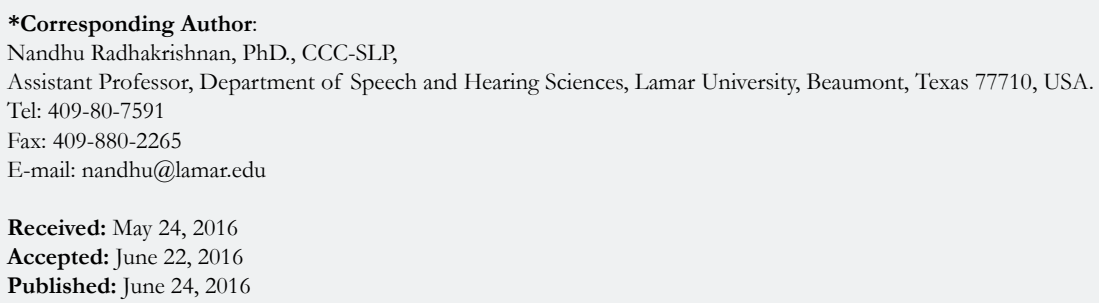

Copyright: Radhakrishnan $\mathbf{N}^{\circ}$ 2016. This is an open-access article distributed under the terms of the Creative Commons Attribution License, which permits unrestricted use, distribution and reproduction in any medium, provided the original author and source are credited. 
Voice analysis including strobolaryngoscopy was performed by the first author. The patient was counseled about the possibilities of change in voice measures. Tailored cricoplasty was performed by the co-author.

\section{Surgical assessment and procedure}

The patient had a $2.5 \mathrm{~cm}$. stenosis involving the cricoid and first 2 tracheal rings. The estimated lumen of the stenosis was $5 \mathrm{~mm}$. The patient underwent resection of the anterior half of the cricoid, the first 3 rings of the trachea. The mucosa overlying the remaining cricoid posteriorly was involved in the inflammatory process and was resected. A posterior membranous wall flap to resurface this defect was created and incorporated into the reconstruction. The recovery was uneventful and discharge occurred on the $8^{\text {th }}$ postoperative day.

The patient was under postoperative care for five months and returned for voice therapy. Postoperative voice was low and hoarse as expected. The patient could neither sing the same notes nor scale the range she had before surgery. CAPE-V, VHI scores worsened.

\section{Voice Therapy}

Conservative voice therapy approach including relaxation, airflow coordination, resonance, and vocal strengthening exercises were followed. After three sessions, the patient did not respond satisfactorily to these exercises. Considering the lowered pitch, principles of transgender voice therapy was used as the primary approach along with modified vocal function exercises (Radhakrishnan \& Scheidt, 2011). Targets and exercises suggested by Adler, Hirsch, and Mordaunt (2006) were adopted. The patient attended therapy twice a week for a total of eighteen 60 minutesessions.

\section{Results}

Voice measures obtained pre-postoperative post-voice therapy is reported in Table-1 and Figures 1-3. Pt showed substantial improvement in mean $\mathrm{F}_{0}$, speaking $\mathrm{F}_{0}$, and perturbation measures. Though phonation frequency range improved after therapy it was less than pre-operative values. Patient satisfaction was reflected in improved VHI-10 scores; however, singing voice continued to be a concern.

Table 1. Voice analysis pre-post operation and post therapy.

\begin{tabular}{|c|c|c|c|c|}
\hline & & Pre-op & Post-op & Post-therapy \\
\hline \multirow{3}{*}{ Subjective } & VHI-10 & 17 & 27 & 9 \\
\cline { 2 - 5 } & CAPE-V & 25 & 30 & 12 \\
\hline \multirow{5}{*}{ Acoustic } & Mean $\mathrm{F}_{0}$ & 221.7 & 176.4 & 205.6 \\
\cline { 2 - 5 } & S.Dev & 3.3 & 8.2 & 2.1 \\
\cline { 2 - 5 } & Speaking $\mathrm{F}_{0}$ & 195.6 & 164.4 & 182.5 \\
\cline { 2 - 5 } & S.Dev & 12.2 & 23.3 & 9.1 \\
\cline { 2 - 5 } & PFR (ST) & 25.3 & 15.4 & 18.5 \\
\cline { 2 - 5 } & Jitter $\%$ & 1.3 & 3.6 & 0.67 \\
\cline { 2 - 5 } & Shimmer \% & 3.4 & 6.04 & 3.46 \\
\hline \multirow{5}{*}{ Aerodynamic } & MPT (s) & 11.2 & 13.5 & 15.1 \\
\cline { 2 - 5 } & Ps cm-water & 6.5 & 5.3 & 4.8 \\
\cline { 2 - 5 } & flow mL/s & 101 & 214 & 150 \\
\hline
\end{tabular}

Figure 1. Comparison of CAPE-V and VHI-10.

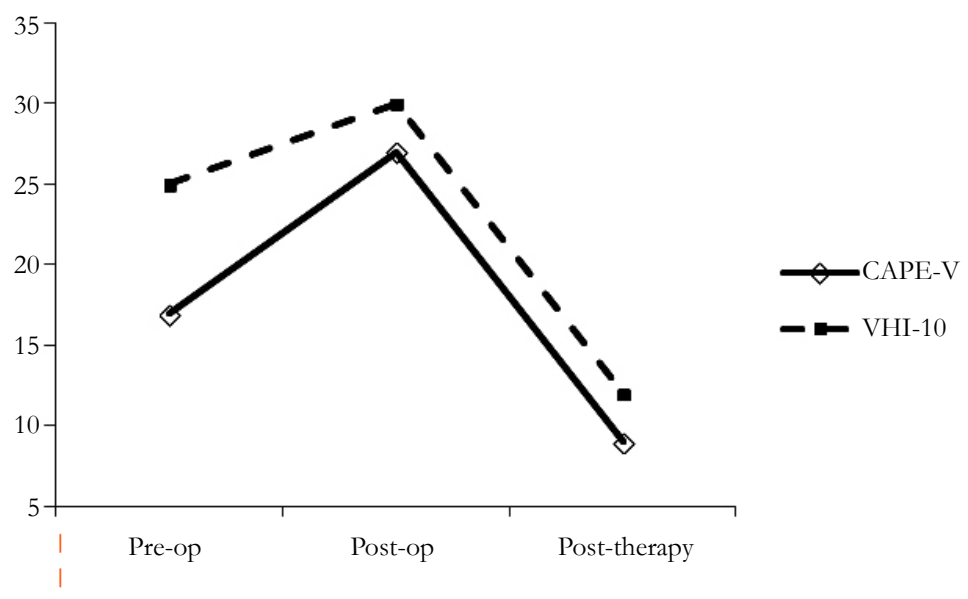


Figure 2. Comparison of Mean $\mathrm{F}_{0}$, Speaking $\mathrm{F}_{0}$, and phonation frequency range.

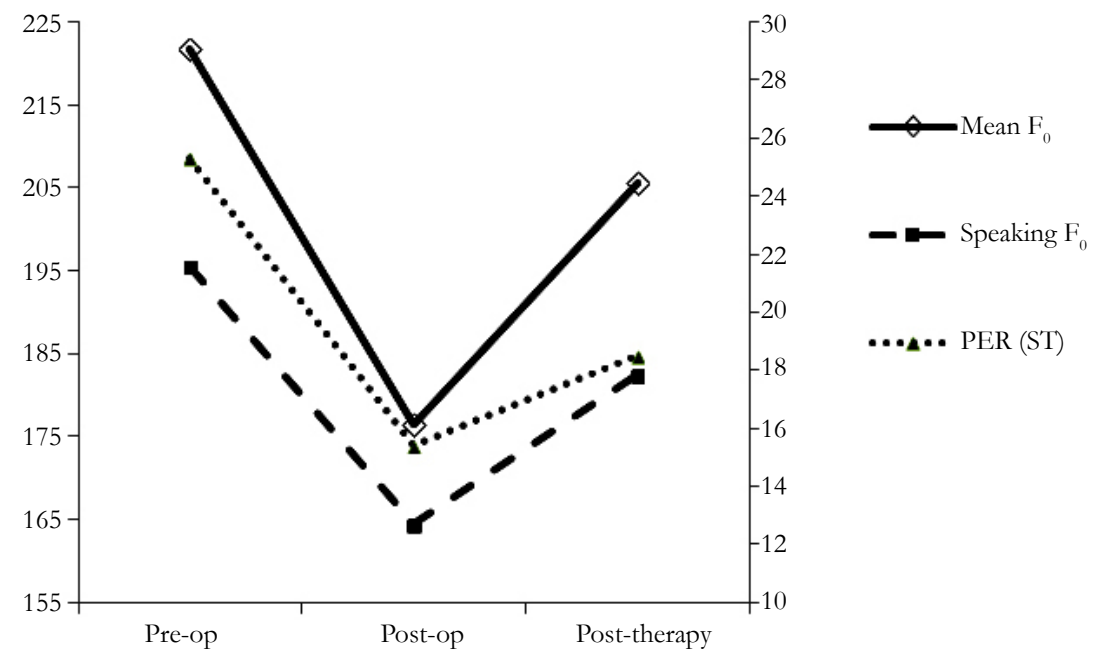

Figure 3. Comparison of perturbation measures, jitter $\%$ and shimmer $\%$.

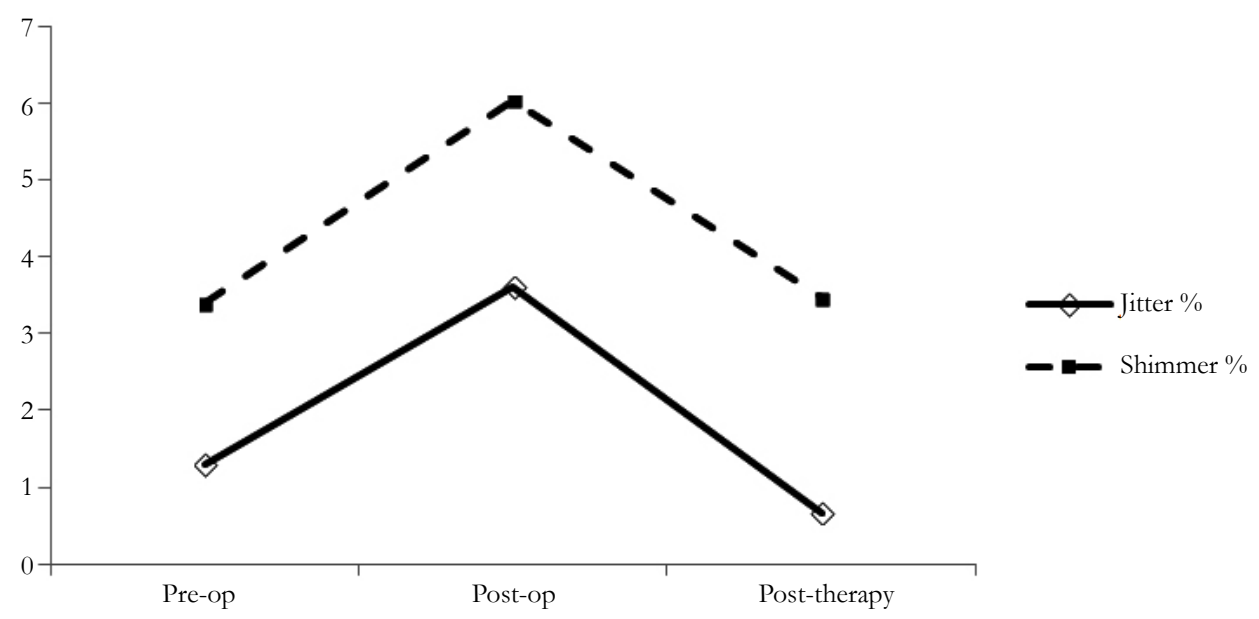

\section{Conclusions}

This study offers impetus to recommend patients with ISS for pre and post-operative voice care. The approach using transgender voice therapy may benefit these patients. The results are based on only one subject; further research using a larger pool of subjects is necessary to learn more about the efficacy of voice therapy in patients undergoing CTR or TC.

\section{Acknowledgements}

The authors would like to thank Ms. Danielle Steel and Ms. Ashley West, graduate student clinicians for working with them in this project.

\section{References}

[1]. Gelbard A, Francis DO, Sandulache VC, Simmons JC, Donovan DT, Ongkasuwan J (2015) Causes and consequences of adult laryngotracheal stenosis. Laryngoscope 125(5): 1137-1143. doi:10.1002/lary.24956
[2]. Valdez TA, Shapshay SM (2002) Idiopathic subglottic stenosis revisited. Annals of Otology Rhinology \& Laryngology 111(8): 690-695.

[3]. Grillo HC, Mathisen DJ, Ashiku SK, Wright CD, Wain JC (2003) Successful treatment of idiopathic laryngotracheal stenosis by resection and primary anastomosis. Annals of Otology, Rhinology \& Laryngology 112(9): 798-800.

[4]. Smith ME, Roy N, Stoddard K, Barton M (2008) How does cricotracheal resection affect the female voice? Annals of Otology Rhinology \& Laryngology 117(2): 85-89.

[5]. Houlton JJ, de Alarcon A, Johnson K, Meinzen-Derr J, Brehm SB, Weinrich B, et al., (2011) Voice outcomes following adult cricotracheal resection. $L a-$ ryngoscope 121(9): 1910-1914.

[6]. Bryans L, Palmer AD, Schindler JS, Andersen PE, Cohen JI (2013) Subjective and objective parameters of the adult female voice after cricotracheal resection and dilation. Annals of Otology Rhinology \& Laryngology 122(11): 707-716.

[7]. Liberman M, Mathisen DJ (2008) Tailored cricoplasty: An improved modification for reconstruction in subglottic tracheal stenosis. Journal of Thoracic and Cardiovascular Surgery 137(3): 573-579. 